\title{
High Level of Suspicion Based on Detailed Medical History and Focused Physical Examination: Important Tool to Diagnose Acute Myocarditis
}

\section{Lahoud Patrick*}

Department of Cardiology, Tel Chiha Hospital, Lebanon \& USEK, Lebanon

*Corresponding author: Lahoud Patrick, Department of Cardiology, Tel Chiha Hospital, Lebanon \& USEK, Lebanon

\begin{abstract}
The present study describes the case of a 39-year-old man who had acute retrosternal pain, history of upper tract infection and low-grade fever 3 days prior to presentation, elevated cardiac markers and electrocardiographic ST-T changes, which led to an original suspicion of acute myocarditis. The patient underwent coronary angiography after 36 hours to rule out coronary artery disease, which revealed normal coronary arteries. Finally, the diagnosis of viral myocarditis was confirmed on consideration of his normal coronary angiography, fever, history of upper tract infection and other auxiliary examination results obtained in the following days, which were supportive of the diagnosis. The patient was managed using NSAID, colchicine, betablocker and angiotensin converting enzymes inhibitors and was discharged 4 days later. Viral myocarditis is a common disease with a variable natural history. It remains challenging for doctors to differentiate between acute myocarditis and myocardial infarction, particularly in the early stages. A diagnosis of myocarditis should be made on the basis of synthetic evaluation of the evidence, including medical history, clinical presentation and results of the available auxiliary tests, in order to provide guidelines for treatment.
\end{abstract}

\section{Introduction}

Myocarditis is inflammation of the myocardium, the cardiac muscle. Acute myocarditis is most commonly caused by viral infections; however, it may less commonly be caused by non-infectious etiologies. Myocarditis can be further divided into acute, subacute, or chronic, and may either affect a focal part of the myocardium or have diffuse involvement. Its clinical presentation may vary from a subtle chest pain or fever, to life-threatening congestive heart failure or dysrhythmia, or even death. Clinical diagnosis is often challenging, and management is usually supportive [1-3]. We report here the initial presentation, medical care, and clinical course of a patient presenting with acute myocarditis.

\section{Case}

A 39-year-old male was admitted to the ER with acute retrosternal pain and tightness that had persisted for $>3 \mathrm{~h}$. Upon presentation, on April 24, 2018, physical examination showed that his blood pressure was $130 / 80 \mathrm{mmHg}$, temperature was $37.7^{\circ} \mathrm{C}$ and heart rate was $79 \mathrm{bpm}$. First and second heart sounds were normal with audible friction rub. No yellow discoloration of the skin or mucous membranes, bleeding or rashes were observed, and the remainder of the examination was normal. No history of hypertension or diabetes was reported, and the patient never smoked cigarettes or drank alcohol.

A series of investigations were subsequently performed in the ER. The results of the cardiac enzyme tests disclosed that the troponin I (TnI) level was significantly elevated, up to $583.2 \mathrm{pg} / \mathrm{ml}$ and CPK $523 \mathrm{IU} / \mathrm{L}$ and CPK-MB $17.47 \mathrm{ng} / \mathrm{ml}$. Of note were the signs of ST-segment elevations in leads V2, V3 and V4 on the electrocardiogram (ECG) (Figure 1). A chest $\mathrm{X}$-ray showed a normal heart size and mild markings in the lungs, with no clear indication of substantive lesion. An echocardiogram showed that the patient's heart functioned normally with an ejection fraction of 


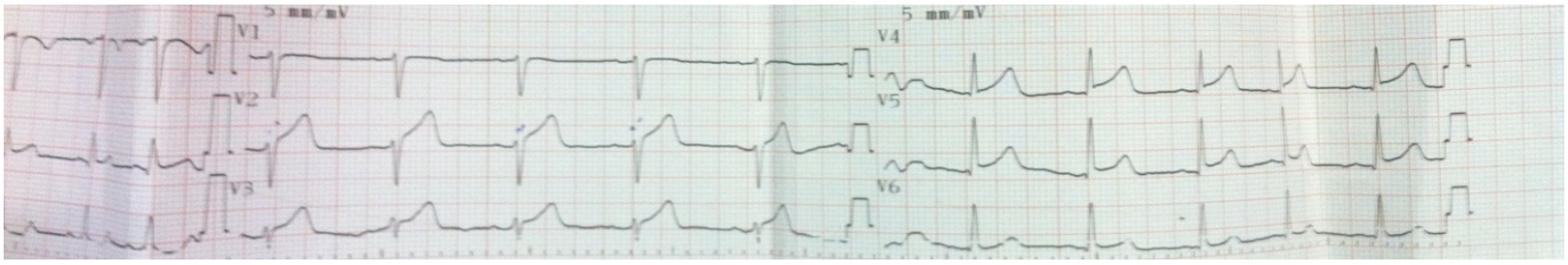

Figure 1: A resting standard electrocardiogram of a 39-year-old Lebanese man. He had ST-segment elevations in leads V2, $\mathrm{V} 3$ and V4.

$57 \%$, excluding the possibility of mitral valve prolapse syndrome, mild AR and mild pericardial effusion behind right ventricle and right atrium.

The patient underwent coronary angiography after 36 hours to rule out coronary artery disease, which revealed normal coronary arteries. A standard 12-lead ECG on the third day showed that the diffuse elevated ST segment began to fall back.

The patient was managed using NSAID, colchicine, betablocker and angiotensin converting enzymes inhibitors and was discharged 4 days later. The clinical course was favorable. A post-discharge 1-month followup visit showed that the patient was recovering well. A holter ECG (24 hours) after 2 months showed normal sinus rhythm without arrhythmias.

\section{Discussion}

Viral myocarditis is a common disease with a variable natural history. In the present case, the patient had retrosternal pain, history of upper tract infection and low-grade fever 3 days prior to presentation, elevated cardiac markers and electrocardiographic ST-T changes on admission. His cardiac enzymes subsequently decreased and the ST-segment elevation gradually decreased. Based on these clinical manifestations and auxiliary examination, the diagnosis of acute myocarditis was confirmed.

Causes of acute myocarditis can be separated into infectious and non-infectious. In $50 \%$ of cases, no cause can be identified; hence, myocarditis is commonly idiopathic. In patients with an identified cause, the most commonly implicated etiology is viral (similar to pericarditis), of which enteroviruses, notably Coxsackie $B$, are the most common. Other viral pathogens include Human Immunodeficiency Virus (HIV), adenovirus, and hepatitis C. Other infectious causes include parasitic (e.g., Trypanosoma cruzi), bacterial (e.g., diphtheria or tuberculosis), helminths, or fungal. In myocarditis related to coronavirus disease 2019 (COVID-19), cardiac inflammation is generally due to direct cardiac invasion with severe acute respiratory syndrome coronavirus 2 (SARS-CoV-2) or a result of the intense cytokine storm that often arises during the course of the disease [4]. Non-infectious causes of myocarditis include granulomatous inflammatory diseases (e.g., sarcoidosis or giant cell myocarditis), systemic lupus erythematosus, Eosinophilic myocarditis, polymyositis and dermatomyositis, and collagen vascular disease [5]. Cases due to cocaine abuse have also been reported in the literature [6].

Given the variable clinical presentation, clinical diagnosis is difficult. The diagnosis is frequently missed and it is difficult to estimate the true incidence of acute myocarditis. However, incidence is estimated to be 10 to 22 per 100,000 cases [7]. It also is estimated that the myocardium is involved in up to $5 \%$ of patients who develop an acute viral illness. Acute myocarditis is more common in younger adults and appears to affect both sexes and various races equally $[8,9]$.

It is believed that myocarditis is largely immune mediated. For example, in infectious etiologies, the microbial agent gains entry through the respiratory or gastroenterology tract and then binds to its specific receptor in the heart. This leads to intracellular replication, resulting in cell damage and lysis. This process may result in immune dysfunction, in which molecular mimicry plays an important role and further enhances cardiac damage. If the damage is severe and prolonged, this can result in dilated cardiomyopathy [10].

The clinical presentation of acute myocarditis is highly variable, ranging from asymptomatic, to subtle to cardiogenic shock and even sudden cardiac death. There are no pathognomonic clinical features. The classic presentation is similar to that of heart failure with symptoms of dyspnea, orthopnea, and leg swelling. Palpitations and syncope may also occur. It is likely that the typical clinical presentations of myocardial infarction, such as chest pain, ST-segment elevation and incremental serum markers, appear in patients diagnosed with myocarditis [11]. In one study of clinically suspected cases, about a quarter of patients had reduced LVEF, sustained ventricular arrhythmias or symptoms of low cardiac output [12]. When the pericardium is involved, as in myopericarditis, chest pain with features similar to pericarditis will arise. Acute myocarditis often presents with a flu-like illness, including fever, myalgia, malaise, nausea and vomiting, for a few days to 3 weeks before any cardiac symptoms appear [12]. The 2013 European Society of 
Cardiology position statement on myocarditis breaks the presentation down to three distinct but overlapping clinical profiles: acute coronary syndrome-like (chest pain not explained by evidence supporting (AD), new onset or worsening heart failure (in the absence of known HF or CAD), and life-threatening conditions (arythmia, cardiogenic shock, severely impaired LV function) [11]. The association of acute myocarditis with ventricular arrhythmias is well established. It has also been recognized that myocardial damage may persist long after the acute phase of myocarditis. Hence, an increased risk for life-threatening arrhythmias seems to be present at any stage of the disease. Longterm follow-up of patients with myocarditis should therefore take into consideration arrhythmic risk stratification, both in patients with preserved and those with reduced left ventricular systolic function [13]. Other manifestations of an infectious agent, such as dysphagia in patients with Chagas disease (caused by Trypanosoma cruzi) or neurological symptoms in patients infected with diphtheria, also may occur. In addition, patients with a non-infectious etiology often will have manifestations of their underlying systemic disease, such as skin or kidney involvement in patients with connective tissue disease.

There are no specific physical examination findings associated with myocarditis. Physical examination may range from normal to features similar to that of heart failure, including S3 gallop, jugular venous distention, peripheral edema, and tachycardia. In addition, patients with ventricular dilatation may have a mitral regurgitation murmur, which is classically described as an apical pansystolic murmur. A pericardial friction rub may be appreciated, especially if there is concurrent pericarditis. Additional findings may suggest underlying disease in non-infectious etiologies. In addition, patients with eosinophilic myocarditis will have a pruritic maculopapular rash [14].

Diagnosis of acute myocarditis is challenging because other clinical entities may mimic the diagnosis. This is further complicated by the variable clinical presentation of acute myocarditis. Acute myocarditis should be suspected in patients with clinical signs and symptoms concerning for the illness regardless of workup, especially in young patients (age 25-50) with no history of cardiac disease. Clues related to underlying etiology may support the diagnosis, such as viral prodrome or signs of connective tissue disease [15-17].

Initial evaluation for acute myocarditis should include an EKG, echocardiogram, serum troponin and BNP. Troponin elevation, which is usually dramatic, is present in more than half of the patients. BNP is useful to assess for evidence heat failure and ventricular stretch, which may suggest myocarditis in the right clinical picture. High concentrations of BNP may correlate with poor prognosis in patients with myocarditis [18]. Caforio, et al. [19] suggested that the log-BNP concentration could be a quantitative biochemical marker of myocarditis in Kawasaki disease. EKG often shows nonspecific ST changes; however, it also may show sinus tachycardia or ventricular arrhythmias (40\% of patients). In addition, changes consistent with pericarditis, including diffuse ST elevation, may be present in those with pericardial involvement. An echocardiogram is useful in assessing the degree of cardiac dysfunction and in helping rule out other causes including valvular disease.

Chest radiograph is neither sensitive nor specific for myocarditis but may show an enlarged heart size, pulmonary vascular congestion or pleural effusion. CT Angiography to evaluate for other causes of chest pain may be indicated. Percutaneus coronary angiography is indicated in patients at high risk for coronary artery disease to help rule out ischemic cause of cardiac dysfunction in the right setting. Imaging with cardiac magnetic resonance seems to be promising and may help differentiate ischemic from non-ischemic etiologies of dilated cardiomyopathy. In addition, a complete blood count with differential showing eosinophilia may hint to eosinophilic myocarditis. Erythrocyte sedimentation rate (ESR) and C-reactive protein (CRP) are usually elevated but non-specific. Viral antibody testing can be done in the right setting; however, specificity is low and often can result in a delay in diagnosis without any change in management.

In recent years, cardiac magnetic resonance imaging (CMRI) has emerged as one of the most useful imaging devices for detecting and diagnosing myocarditis, as it can provide various means to visualize and quantify myocardial inflammatory changes [20,21]. Furthermore, late gadolinium enhancement (LGE) quantification and pattern of distribution, as well as abnormal T1 mapping and extracellular volume (ECV), representing indices of diffuse fibrosis, can enhance our ability to detect high risk patients. Cardiac magnetic resonance can also complement electro-anatomical mapping (EAM), a technique already applied in the risk evaluation and in the ventricular arrhythmias ablation therapy of CM patients, providing a more accurate assessment of fibrosis and arrhythmic corridors. As a result, cardiac magnetic resonance provides a new insight into the pathological substrate of cardiomyopathies (CM). Cardiac magnetic resonance imaging may help identify high risk CM patients and, combined with EAM, can provide an integrated evaluation of scar and arrhythmic corridors in the ablative therapy of ventricular arrhythmias [22]. A total of 78 patients who recovered from COVID-19 infection (78\%) had cardiovascular involvement as detected by standardized $\mathrm{CMR}$, irrespective of preexisting conditions, the severity and overall course of the COVID-19 presentation, the time from the original diagnosis, or the presence of cardiac symptoms. The most prevalent abnormality was myocardial inflammation (defined as abnormal 
native T1 and T2 measures), detected in 60 patients recently recovered from COVID-19 (60\%), followed by regional scar and pericardial enhancement [23]. CMRI, however, is not accepted by a proportion of patients with suspected myocarditis due to the considerable expense. The current patient was one such case.

Finally, the histological and immunological evaluation of biopsies can be used in the diagnosis of acute myocarditis. Not infrequently, patients presenting with ventricular arrhythmias and no apparent structural cardiac abnormalities, may have underlying myocardial inflammation, as previously shown in endomyocardial biopsy studies [24]. Endomyocardial biopsy (EMB) is not a routine diagnostic method in the majority of cases of suspected acute myocarditis, since it is an invasive approach and has a probability of sampling error due to the characteristic patchy inflammation and variability in observer interpretation. A 2013 position statement from the European Society of Cardiology Working Group on Myocardial and Pericardial Diseases [19] recommended heart biopsy as a routine test for all cases of suspected myocarditis. Conversely, the routine application of EMB was not recommended by the 2013 American College of Cardiology Foundation/American Heart Association [25]; therefore, no consensus has been reached with regard to the application of EMB in the diagnosis of myocarditis. EMB was not performed in the present case.

According to the Canadian Cardiovascular Society Consensus Conference guidelines on heart failure (updated 2009), EMB evaluation for myocarditis should include the use of histopathological markers of inflammation and necrosis, immunohistochemical markers and the assessment of viral particles [26]; however, it has been indicated that the histological diagnosis of myocarditis based on the Dallas criteria lacks sensitivity and specificity [27]. Additionally, an absence of sensitive markers for an active immunological process can limit the use of histopathological analysis [28]. Immunohistochemical techniques can enable the quantification and identification of activated inflammatory cells, including $\mathrm{T}$ lymphocytes, B cells, macrophages and natural killer cells. Among those cells, T lymphocytes are essential for diagnosing active myocarditis. Immunostains for cell-specific markers may also help confirm the presence of myocardial inflammation. The analysis of viral replication in the myocardium through the use of the polymerase chain reaction and in situ hybridization can help quantify the specific viral variants accounting for myocardial damage. Previous findings using these novel diagnostic tests point toward a broader spectrum of viral genomes responsible for acute myocarditis, indicating a shift from enterovirus and adenovirus to parvovirus B19 and human herpes-virus 6 as the viruses most frequently causing acute myocarditis [29-31].
Management is primarily supportive but also includes treating any identifiable cause. $\sim 50 \%$ of the patients with acute myocarditis are likely to spontaneously recover within a month, $\sim 25 \%$ will develop persistent impaired cardiac function and up to $25-30 \%$ may either progress to dilated cardiomyopathy, making heart transplantation a necessity, or succumb to the condition [31,32].

Patients with heart failure should receive standard treatment including beta-blockers, angiotensinconverting enzyme inhibitors or angiotensin II receptor blockers, and diuretics if needed. Immunosuppressive therapy has not shown clinical benefit and therefore should not be used routinely except in patients who have underlying systemic autoimmune or granulomatous inflammatory diseases. Since patients are generally diagnosed with myocarditis within days or weeks after the initial viral infection, antiviral therapy is seldom used in clinical practice in the early phase of myocarditis, although antiviral therapy has been reported to have a positive effect in the acute viremic stage [33]. A patient with parvovirus-B19-associated fulminant myocarditis was reported to show a complete recovery with immunosuppressive and antiviral therapy (intravenous immunoglobulin and acyclovir) within 2 weeks [34]. Furthermore, the antiviral effect of interferon- $\beta$ therapy, enhanced by the transcription suppressor 4E-BP1, has been reported in myocarditis induced by coxsackievirus B3 [35].

Immune suppression may be beneficial in patients with systemic disease-related or autoimmune myocarditis but may increase virus replication and worsen myocardial injury in viral myocarditis. A study showed that immunosuppressive treatment taken immediately on detection of sclerotic heart disease proved effective in preventing cardiac damage progression [36] Similarly, an improved performance of immunosuppressive therapy (azathioprine and prednisone) was reported for children with chronic myocarditis, regardless of the presence of viral infection, compared with conventional therapy [37]. By contrast, Hia, et al. [38] reviewed the impact of immunosuppressive therapy on the outcome of acute myocarditis in children, and the 18 years of data suggested that immunosuppressive therapy does not significantly improve outcomes in children with acute myocarditis, providing negative evidence for its routine use [38]. It is therefore necessary to evaluate the type of myocarditis carefully prior to starting the immunosuppressive therapy in order to avoid its ill effects.

Non-steroid anti-inflammatory agents should be avoided in the acute setting as they may impair healing of the myocardium. In the present case, due to severe retrosternal pain and mild pericardial effusion NSAID and colchicine was administered. In severe cases, mechanical support devices such as an intra-aortic pump 
or left ventricular assist devices also can be used [39] with consideration of heart transplant as well. In these instances, it is important to consider a transfer to tertiary care centers for surgical support if needed. Patients may develop dysrhythmias or left atrial or ventricular thrombus requiring anticoagulation. If patients present with severe refractory ventricular arrhythmias or atrioventricular blocks, they may require antiarrhythmic medication or the insertion of implantable cardioverter defibrillators or temporary pacemakers, respectively [28]. Finally, patients should be counseled on limiting exercise and avoiding alcohol, especially in the acute phase, as it may increase viral replication. Long-term follow-up with serial echocardiography is recommended $[40,41]$.

\section{Conclusion}

Despite considerable progress, it remains a challenge for physicians to discriminate between acute myocarditis and myocardial infarction, particularly in the early phase. An integrated assessment and evaluation of evidence, including medical history, clinical presentation and results of other auxiliary tests, are necessary for the accurate diagnosis of myocarditis and can guide treatment accordingly. In the current case, a patient with viral myocarditis presented with retrosternal pain, elevated cardiac marker levels and ST-T changes on the ECG, similar to the clinical manifestations of acute myocardial infarction. Clinical manifestations, including a fever, coronary angiography and the results of auxiliary examinations, could aid in the differential diagnosis between acute myocarditis and myocardial infarction. The etiology, pathology, diagnostics and therapy of myocarditis remain controversial. Future investigations are required to further unravel these questions.

\section{References}

1. Mehta JJ, Vindhyal MR, Boppana VS, Farhat A (2018) Focal myopericarditis presenting as acute ST-elevation myocardial infarction. Kans J Med 11: 83-85.

2. Jois $A$, Zannino D, Curtis N, Cheung M, Burgner DP, et al. (2019) Arterial structure and function following viral myocarditis. Pediatr Cardiol 40: 133-137.

3. Mert GO, Radi F, Mert KU (2018) The diagnostic challenge: Takotsubo cardiomyopathy vs. acute myocarditis. Heart Lung 47: 646.

4. Rezkalla SH, Kloner RA (2021) Viral myocarditis: 19172020: From the Influenza A to the COVID-19 pandemics. Trends Cardiovasc Med 31: 163-169.

5. Takeuchi S, Kawada JI, Okuno Y, Horiba K, Suzuki T, et al. (2018) Identification of potential pathogenic viruses in patients with acute myocarditis using next-generation sequencing. J Med Virol 90: 1814-1821.

6. Dionne A, Dahdah N (2018) Myocarditis and Kawasak disease. Int J Rheum Dis 21: 45-49.

7. Chang JJ, Lin MS, Chen TH, Chen DY, Chen SW, et al. (2017) Heart failure and mortality of adult survivors from acute myocarditis requiring intensive care treatment - a nationwide cohort study. Int J Med Sci 14: 1241-1250.
8. Baessler B, Luecke C, Lurz J, Klingel K, von Roeder M, et al. (2018) Cardiac MRI texture analysis of T1 and T2 maps in patients with infarctlike acute myocarditis. Radiology 289: 357-365.

9. Leong K, Kane JM, Joy BF (2018) Acquired cardiac disease in the pediatric intensive care unit. Pediatr Ann 47: e280-e285.

10. Datta T, Solomon AJ (2018) Clozapine-induced myocarditis. Oxf Med Case Reports 2018: omx080.

11. Abutaleb ARA, McNally EM, Khan SS, Anderson AS, Carr JC, et al. (2018) Myocarditis in duchenne muscular dystrophy after changing steroids. JAMA Cardiol 3: 10061010.

12. Saricam E, Saglam Y, Hazirolan T (2017) Clinical evaluation of myocardial involvement in acute myopericarditis in young adults. BMC Cardiovasc Disord 17: 129.

13. Gatzoulis KA, Archontakis S, Vlasseros I, Tsiachris D, Vouliotis A, et al. (2014) Complex right ventricular outflow tract ectopy in the absence of organic heart disease. Results of a long-term prospective observational study. Int J Cardiol 172: e351-e353.

14. Bigalke B, Klingel K, May AE, Kandolf R, Gawaz M (2007) Human herpes virsus 6 subtype A-associated myocarditis with 'apical ballooning'. Can J Cardiol 23: 393-395.

15. Maisch B, Alter P (2018) Treatment options in myocarditis and inflammatory cardiomyopathy: Focus on i. v. immunoglobulins. Herz 43: 423-430.

16. Godishala A, Yang S, Asnani A (2018) Cardioprotection in the modern era of cancer chemotherapy. Cardiol Rev 26: 113-121.

17. Zheng YM, Yang J, Liao QH (2017) Health related quality of life on severe hand, foot and mouth disease patients. Zhonghua Liu Xing Bing Xue Za Zhi 38: 462-466.

18. Zhang C, Shen D, Sun H, Zhang L, Ma Y, et al. (2012) Prognostic value of brain natriuretic peptide in people with viral myocarditis. Zhonghua Shi Yan He Lin Chuang Bing Du Xue Za Zhi 26: 125-126.

19. Caforio ALP, Pankuweit S, Arbustini E, Basso C, GimenoBlanes J, et al. (2013) Current state of knowledge on aetiology, diagnosis, management and therapy of myocarditis: A position statement of the european society of cardiology working group on myocardial and pericardial diseases. Eur Heart J 34: 2636-2648.

20. Olimulder M, Van Es J, Galjee MA (2009) The importance of cardiac MRI as a diagnostic tool in viral myocarditisinduced cardiomyopathy. Neth Heart J 17: 481-486.

21. Chan A, Tan T, Sosnovik D, Francis S, Ghoshhajra B (2014) Acute viral myocarditis diagnosis by cardiac magnetic resonance imaging. Cardiovascular Images: 58.

22. Kariki O, Antoniou CK, Mavrogeni S, Gatzoulis KA (2020) Updating the risk stratification for sudden cardiac death in cardiomyopathies: The evolving role of cardiac magnetic resonance imaging. An approach for the electrophysiologist. Diagnostics (Basel) 10: 541.

23. Puntmann VO, Carerj ML, Wieters I, Fahim M, Arendt C, et al. (2020) Outcomes of cardiovascular magnetic resonance imaging in patients recently recovered from coronavirus disease 2019 (covid-19). JAMA Cardiol 5: 1265-1273.

24. Strain JE, Grose RM, Factor SM, Fisher JD (1983) Results of endomyocardial biopsy in patients with spontaneous ventricular tachycardia but without apparent structural heart disease. Circulation 68: 1171-1181. 
25. Yancy CW, Jessup M, Bozkurt B, Butler J, Casey DE, et al. (2013) ACCF/AHA guideline for the management of heart failure: A report of the American college of cardiology foundation/american heart association task force on practice guidelines. J Am Coll Cardiol 62: e147-e239.

26. Howlett JG, McKelvie RS, Arnold JM, Costigan J, Dorian P, et al. (2009) Canadian Cardiovascular Society Consensus Conference guidelines on heart failure, update 2009: Diagnosis and management of right-sided heart failure, myocarditis, device therapy and recent important clinical trials. Can J Cardiol 25: 85-105.

27. Baughman KL (2006) Diagnosis of myocarditis: Death of Dallas criteria. Circulation 113: 593-595.

28. Kuhl U, Schultheiss HP (2009) Viral myocarditis: Diagnosis, aetiology and management. Drugs 69: 1287-1302.

29. Dennert R, Crijns HJ, Heymans S (2008) Acute viral myocarditis. Eur Heart J 29: 2073-2082.

30. Basso C, Calabrese F, Angelini A, Carturan E, Thiene G (2013) Classification and histological, immunohistochemical and molecular diagnosis of inflammatory myocardial disease. Heart Fail Rev 18: 673-681.

31. Bock CT, Düchting A, Utta F, Brunner E, Tien Sy B, et al. (2014) Molecular phenotypes of human parvovirus B19 in patients with myocarditis. World J Cardiol 6: 183-195.

32. Kindermann I, Barth C, Mahfoud F, Ukena C, Lenski M, et al. (2012) Update on myocarditis. J Am Coll Cardiol 59: 779-792.

33. Gupalo E, Mironova N, Stukalova O, Zykov K, Buryachkovskaya L, et al. (2013) A case of arrhythmia due to myocarditis treated by antiviral therapy: New diagnostic approaches using peripheral biomarkers. Eur Heart $\mathrm{J} 34$ 17.
34. Dennert R, Velthuis $S$, Westermann D, Donker D, Schalla S, et al. (2010) Case report Parvovirus-B19associated fulminant myocarditis successfully treated with immunosuppressive and antiviral therapy. Antivir Ther 15: 681-685.

35. Burke JD, Sonenberg N, Platanias LC, Fish EN (2011) Antiviral effects of interferon- $\beta$ are enhanced in the absence of the translational suppressor 4E-BP1 in myocarditis induced by Coxsackievirus B3. Antivir Ther 16: 577-584.

36. Pieroni M, De Santis M, Zizzo G, Bosello S, Smaldone C, et al. (2014) Recognizing and treating myocarditis in recent-onset systemic sclerosis heart disease: Potential utility of immunosuppressive therapy in cardiac damage progression. Semin Arthritis Rheum 43: 526-535.

37. Camargo PR, Okay TS, Yamamoto L, Del Negro GM, Lopes AA (2011) Myocarditis in children and detection of viruses in myocardial tissue: Implications for immunosuppressive therapy. Int J Cardiol 148: 204-208.

38. Hia CP, Yip WC, Tai BC, Quek SC (2004) Immunosuppressive therapy in acute myocarditis: An 18 year systematic review. Arch Dis Child 89: 580-584.

39. Grinda J-M, Chevalier P, D'Attellis N, Bricourt M-O, Berrebi A, et al. (2004) Fulminant myocarditis in adults and children: Bi-ventricular assist device for recovery. Eur J Cardiothorac Surg 26: 1169-1173.

40. Veronese G, Ammirati E, Cipriani M, Frigerio M (2018) Fulminant myocarditis: Characteristics, treatment, and outcomes. Anatol J Cardiol 19: 279-286.

41. Global Burden of Disease Study 2013 Collaborators (2015) Global, regional, and national incidence, prevalence, and years lived with disability for 301 acute and chronic diseases and injuries in 188 countries, 1990-2013: A systematic analysis for the Global Burden of Disease Study 2013. Lancet 386: 743-800. 